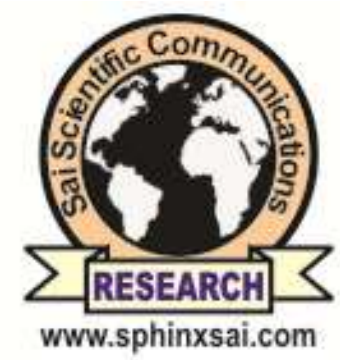

\title{
Acne:Clinico-Epidemiological Study In Dermatology- Venereology Outpatient Clinic Of Dr. M. Djamil Hospital Padang During January 2016 - December 2018
}

\author{
Satya Wydya Yenny \\ ${ }^{1}$ Dermatology and Venereology Department of Dr.M.Djamil General Hospital \\ Medical Faculty of Andalas University. Padang/Indonesia Telephone : 08126617869
}

\begin{abstract}
Although acne is usually recognized as an adolescent skin disorder, the prevalence of adults with acne is increasing. The clinical and epidemiological data of acne were evaluated with a view to establishing possible contributing etiological factors and observing whether clinical features differ from adolescent acne. Division of Dermatology and Venereology Outpatient Clinic Dr. M. Djamil hospital padang during January 2016 until December 2018.Retrospective study performed in Medical Cosmetic Division of Dermatology and Venereology Outpatient Clinic Dr. M. Djamil hospital padang during January 2016 until December 2018. Data was taken from medical records. Out of 224 patients included in the study $54.01 \%$ were women and $45.98 \%$ were men. Majority of the patients had comedonal acne $(45.53 \%)$, whereas nodulocystic was the least common (13.39\%). Most common predominant site of involvement was cheek (44.20\%), followed by chin (25.45\%), and mandibular area $(14.58 \%)$. Family history of acne was present in $57.70 \%$. Scarring was observed in a $39.2 \%$. Acne is predominant in women, with the most commonly involved of the cheeks, with the most common type was comedones type.
\end{abstract}

Keyword : Acne, epidemiology, Indonesia, profile.

\section{Introduction}

Acne is a common skin disease that affects nearly $80 \%$ of adolescents and young adults aged 11 to 30 years. ${ }^{1-4}$ Acne can present at any age, affecting $8 \%$ of adults aged 25 to 34 years and $3 \%$ of those aged 35 to 44 years. Lesions appear primarily on the face, back, chest, and other areas with a high concentration of pilosebaceous glands. ${ }^{1}$

Various clinical presentations include seborrhea, comedones, erythematous papules and pustules, less frequently nodules, deep pustules or pseudocysts and ultimate scarring in few of them. Acne has a negative effect on the quality of life; although this can be improved with effective treatment. ${ }^{1}$

Acne has four main pathogenic mechanisms: increased sebumproduction, hyperkeratinization, propionibacterium acne colononization and the inflammatory reaction. Therapeutic modalities are designed now due to better understanding of the pathogenesis of acne. ${ }^{1}$ 


\section{Methods:}

The present study is retrospective study using medical records patients with acne in Dr. M. Djamil hospital Padang between January 2016 - December 2018. The medical records consist of age, sex, family history of acne, duration of disease, distribution of lesions, precipitating factors and type of acne.

\section{Result}

The study included 224 patients, 121 (54.01\%) women and 103 (45.98\%) men. The incidence of acne in this study was found to be $0.4 \%$.

Table.1. Prevalence of adult acne

\begin{tabular}{|c|c|c|}
\hline Year & $\begin{array}{c}\text { Case Number } \\
(\mathbf{N}=\mathbf{2 2 4})\end{array}$ & Percentage total case \\
\hline 2016 & 82 & $36.61 \%$ \\
\hline 2017 & 75 & $33.48 \%$ \\
\hline 2018 & 67 & $29.91 \%$ \\
\hline
\end{tabular}

The most common age of the patients was $15-<24$ years. The proportion of population of different age groups is detailed in table 2 . The most common patient had a family history of acne, $127(56,7 \%)$.

Table 2. Characteristic of Acne Patients in Dermatology-Veneorology Outpatient Clinic of Dr. M. Djamil Hospital Padang during January 2016 - December 2018

\begin{tabular}{|c|c|c|}
\hline $\begin{array}{c}\text { Epidemiology and clinical } \\
\text { profile }\end{array}$ & $\begin{array}{c}\text { Case number } \\
(\mathbf{n = ~ 2 2 4 )}\end{array}$ & $\begin{array}{c}\text { Percentage Total Cases } \\
(\mathbf{\%})\end{array}$ \\
\hline Sex & & $45.98 \%$ \\
\hline Male & 103 & $54.01 \%$ \\
\hline Female & 121 & $56.70 \%$ \\
\hline Age (Year) & 127 & $32.14 \%$ \\
\hline $15-<24$ & 72 & $11.16 \%$ \\
\hline $24-<44$ & 25 & $0 \%$ \\
\hline $44-<64$ & 0 & $38.39 \%$ \\
\hline$\geq 64$ & & $25 \%$ \\
\hline Duration of acne (months) & 86 & $16.52 \%$ \\
\hline $0-12$ & 56 & $8 \%$ \\
\hline $13-24$ & 37 & $6.25 \%$ \\
\hline $25-36$ & 18 & $5.8 \%$ \\
\hline $37-48$ & 14 & \\
\hline $49-60$ & 13 & $56.70 \%$ \\
\hline$>60$ & & $33.93 \%$ \\
\hline Family History & 127 & \\
\hline Yes & 97 & \\
\hline No & & \\
\hline
\end{tabular}

Table 3. Clinical characteristic

\begin{tabular}{|c|c|c|}
\hline Clinical Characteristic & n & \% \\
\hline Severity of acne & & \\
\hline Mild & 88 & $39.28 \%$ \\
\hline Moderate & 75 & $33.48 \%$ \\
\hline Severe & 61 & $27.23 \%$ \\
\hline Post acne scarring & & \\
\hline
\end{tabular}




\begin{tabular}{|c|c|c|}
\hline Yes & 88 & $39.2 \%$ \\
\hline No & 136 & $60.71 \%$ \\
\hline Distribution of lesions & & \\
\hline Cheek & 99 & $44.20 \%$ \\
\hline Forehead & 23 & $10.27 \%$ \\
\hline Mandibular & 32 & $14.28 \%$ \\
\hline Chin & 57 & $25.45 \%$ \\
\hline Nose & 13 & $5.80 \%$ \\
\hline Type of Acne & & \\
\hline Comedonal & 102 & $45.53 \%$ \\
\hline Papulopustul & 92 & $41.07 \%$ \\
\hline Nodulocystic & 30 & $13.39 \%$ \\
\hline
\end{tabular}

A total of 224 patients, 88 (39.28\%) had mild acne vulgaris, and $75(33.48 \%)$ had moderate acne. Severe of acne vulgaris was diagnosed in 61 patients $(27.23 \%)$. Post-acne scarring was seen in 88 patients (39.2\%). The most common type of acne in this study was comedones $(45.53 \%)$. Followed by papulopustul $(41.07 \%)$ and nodulocystic (13.39\%). Post acne scarring was $88(39.2 \%)$.

\section{Discussion}

Acne vulgaris is a chronic condition that is virtually universal in adolescence. An individual is more likely to develop acne than any other disease. Hospital-based studies done on acne vulgaris in Asian population have shown that acne vulgaris constitutes $11.2 \%$ and $19.6 \%$ of the total new patients attending their hospitals. A survey of Australian private dermatology practices reported that of 3197 new diagnoses, $320(10 \%)$ patients were for acne. In our study, The study included 224 patients, $121(54.01 \%)$ women and $103(45.98 \%)$ men. The incidence of acne in this study was found to be $0.4 \%$. The most common age of the patients was $15-<24$ years. The greater age in our population may be associated with a delay in consulting a dermatologist. The marked female predominance in our case series was also reflected in a French study,by contrast with other investigations which have reported more cases in boys. ${ }^{2}$ Al-Ameer and Al-Akloby, in their study of 225 patients with acne vulgaris observed that the age at presentation was $19.2 \pm 3.0$ years for males and $18.4 \pm 4.2$ years for females. Kane et al.noted that the mean age of presentation of their patients was 25.58 years. ${ }^{3}$ Epstein established a prevalence of $42 \%$ of women with acne aged between 26 and 45 years. In a more recent study conducted by Goulden et al., the prevalence of acne in a community-based population of adults, clinical facial acne was assessed in $12 \%$ of women and $3 \%$ of men. When both physiological and clinical acne were assessed, facial acne was observed in $54 \%$ of women and $40 \%$ of men. ${ }^{4}$

It leaves scarring, which was observed in $39.2 \%$ of our patients. By contrast, scarring was reported in only $25 \%$ by Kilkenny et al. and $5.9 \%$ by Susan et al. Chi keung yeung et.al (Hong Kong, 2002) was reported $52.6 \%$ of subjects had acne scarring. ${ }^{5}$ V. Goulden (United Kingdom, 1999), acne scarring was recorded in $12 \%$ of volunteers. ${ }^{6}$ Niti Khunger et al ( India, 2012), reported acne scarring was observed in a majority of patients (76.4\%). Department This is an expected finding because acne scarring has been shown to correlate with the duration of acne. Scarring in adult acne is more common as compared to adolescent acne. This is probably because acne in adults is predominantly inflammatory, may be resistant to treatment and treatment is often delayed, which leads to greater scarring. Scarring can occur as a result of damage to the skin during the healing of active acne. There are two basic types of scar depending on whether there is a net loss or gain of collagen (atrophic and hypertrophic scars). Eighty to ninety percent of people with acne scars have scars associated with a loss of collagen (atrophic scars) compared to a minority who show hypertrophic scars and keloids. ${ }^{7}$

In this study, the cheek was the most common site on face in comparison to involvement of mandibular area. This is in contrast to studies from western countries that have reported involvement of lower face and mandibular area more commonly in adult acne cases. Acne vulgaris is a polymorphic disease. The primary and the pathognomonic lesion of acne vulgaris is a comedone, which may be open or closed. Kilkenny et al. and 
Cunliffe et al. reported that comedones were the most common type of lesion. It is known that the closed comedones are usually present in much greater numbers than open comedones.

It has been suggested that acne might be familial according to the results of a large study of identical twins showing $97.9 \%$ of affection. In our study population, $56.7 \%$ patients had at family history of acne, while Goulden et al., observed a higher incidence of 50\%. Hence it seems to be true that genetic factors might play role in acne development and persistence.

\section{Conclusion}

The incidence of Acne in Dr. M. djamil hospital was $0.4 \%$. The most commonly affected was young adult. Comedonal was the most common type of acne. Majority of the patients had mild severity of acne. Post acne scarring was the most commonly affected in this study. Majority of the patients in this study had family history of acne.

\section{Acknowledgement}

None

\section{References}

1. Seth V, Mishra A, Seth V, Med JA. Acne vulgaris management: what's new and what's still true? 2015;2(1):1-5.

2. Kane A, Niang SO, Diagne AC, Ly F, Ndiaye B. Epidemiologic, clinical, and therapeutic features of acne in Dakar, Senegal. 2007;(December 2002):36-38.

3. Adityan B, Thappa DM. Pro P le of acne vulgaris - A hospital-based study from. 2009;75(3).

4. Poli F. An epidemiological study of acne in female adults : results of a survey conducted in France. 2014;(2001):541-545.

5. $\quad$ Movita T. Acne Vulgaris. 2013;40(3):269-272.

6. G V, Stables GI. Prevalence of facial acne in adults. 1999;(March 1996):577-580.

7. Fabbrocini G, Annunziata MC, D’Arco V, et al. Acne scars: Pathogenesis, classification and treatment. Dermatol Res Pract. 2010;2010(1). 